\title{
EFFECT OF DIFFERENT VOXEL SIZES ON ACCURACY OF 2D AND 3D MANDIBULAR LINEAR MEASUREMENTS USING CRANEX® 3D CONE BEAM COMPUTED TOMOGRAPHY MACHINE: PRECLINICAL STUDY
}

\author{
Mohamed Selim*, Mushira Abdel Latif Dahaba ${ }^{* *}$ and Ahmed Abdalsamad ${ }^{* * *}$
}

\begin{abstract}
Aim To assess the accuracy of linear measurement obtained from CBCT images in 3D volumetric rendering and multiplanar slices with different voxel sizes.

Methodology: 9 mandibles with twelve radioopaque markers fixed on each one were imaged using CRANEX® 3D CBCT machine. Each mandible was imaged twice with voxel sizes 250 $\mu \mathrm{m}$ and $350 \mu \mathrm{m} .2 \mathrm{D}$ and 3D linear measurements from CBCT images were compared to the real physical measurements taken by a digital caliper. Software measurements were recorded twice by the principal investigator and once by the supervising professor. Intra- and inter-observer agreements were assessed using Intra-Class Correlation Coefficient (ICC). SPSS®IBM Version 24 and MedCalc ${ }^{\circledR}$ version 12.1 for Windows were used for statistical analysis.
\end{abstract}

Results: CBCT 2D measurements with voxel sizes of $250 \mu$ and $350 \mu$ showed a mean difference $=0 \mathrm{~mm}$ both with $95 \%$ of difference being $-0.6 \mathrm{~mm}$ above or $0.7 \mathrm{~mm}$ below and $-0.5 \mathrm{~mm}$ above or $0.6 \mathrm{~mm}$ below the Gold Standard respectively. CBCT 3D measurements with voxel sizes of $250 \mu$ and $350 \mu$ showed a mean difference $=0 \mathrm{~mm}$ both with $95 \%$ of difference being $-0.5 \mathrm{~mm}$ below or $0.5 \mathrm{~mm}$ above and $-0.6 \mathrm{~mm}$ above or $0.7 \mathrm{~mm}$ below the Gold Standard. Intra-observer and inter-observer reliability regarding all measurements showed excellent agreement.

Conclusions: 2D and 3D measurements driven from CBCT images with voxel size of $350 \mu$ are accurate as measurements driven from CBCT images with voxel size of $250 \mu$ with less radiation delivered to the patient. 2D and 3D CBCT measurements driven from CBCT are reliable.

KEYWORDS: CBCT, Linear measurements, Voxel

* Oral Radiology Department, Faculty of Dentistry, Cairo University, Cairo, Egypt

** Professor, Oral and Maxillofacial Radiology Department, Cairo university- Giza - Cairo

*** Assistant Professor, Radiology Faculty of Dentistry Cairo University 


\section{INTRODUCTION}

Cone Beam Computed Tomography (CBCT) is a technology that is needed to overcome the limitations of the 2D imaging modalities as periapical, panoramic and lateral cephalometric radiography because of the three dimensional nature of the scanned object ${ }^{(1,10)}$.

CBCT can perform imaging of maxillofacial structures with different voxel sizes considering that smaller voxel size provides better image resolution but requires higher radiation dose $\mathrm{e}^{(19,20)}$.

CBCT is used in oral and maxillofacial surgery for surgical evaluation and planning for orthognathic, implant and maxillofacial surgeries and demonstrating craniofacial fractures ${ }^{(3,18,21)}$. Moreover, it is used in endodontics as in detection of missed canals and root fractures ${ }^{(11,12)}$.CBCT was found better than conventional radiography in the detection of longitudinal fractures ${ }^{(2)}$. In periodontics it is used in the detection of intra-bony and furcation defects, dehiscence, fenestration and periodontal cysts ${ }^{(14,16)}$.

The precision of the jaw measurements is important for many of the aforementioned dental procedures. It's of vital importance to know the measurement accuracy and reliability of $\mathrm{CBCT}^{(17)}$. There are two main types of linear measurements that could be obtained from CBCT scans, the 2D linear measurement using 2D ruler on multiplanar slices and 3D linear measurement using 3D ruler on $3 \mathrm{D}$ reconstructed image ${ }^{(4,6)}$. That's why this study was undertaken to assess the accuracy of $2 \mathrm{D}$ and $3 \mathrm{D}$ linear mandibular measurements in CBCT images taken with 2 voxel sizes: $0.25 \mathrm{~mm}$ and $0.35 \mathrm{~mm}$.

\section{MATERIALS AND METHODS}

\section{Study Design}

The study was performed on 9 human dry mandibles acquired from the Anatomy Department, Faculty of Medicine, Cairo University, Kasr El Einy Hospital.The selected mandibles were intact, free from any bone defects, fractures, pathology. Dentate state of the mandibles was not an element in selection criteria; either dentulous or edentulous mandibles were acceptable.

\section{Mandibles Preparation and Real Measurements (Gold Standard):}

Twelve points were marked on the mandibles and radio opaque markers (Uniform cylindrical shapes with standardized size made of Barium Sulphate mixed with clear resin) were glued to each mandible at these points using wax and double face tape. The points were assigned capital letters (A, B, C, D, E, F, G, H, I, $\mathrm{J}, \mathrm{K} \& \mathrm{~L}$ ) distributed as illustrated in Figure 1.Twelve Connecting lines were decided to be measured as follows: AB, AE, AJ, KI, FD, JL, EG, HC, JE, GL, BL and BG.First the real physical measures were taken by a digital caliper between the points.

\section{Soft Tissue Simulation:}

The mandibles were placed in a plastic container filled with water and totally immersed to simulate the soft tissue attenuation

\section{CBCT Imaging of the Mandibles:}

CBCT imaging was performed in a private radiology center [Orascan] using CRANEX ${ }^{\circledR}$ 3D (SoredexOy, Tuusula, Finland) CBCT machine. Each mandible was imaged twice with voxel sizes $250 \mu \mathrm{m}$ and $350 \mu \mathrm{m}$ using the following exposure parameters: $(250 \mu \mathrm{m}$ voxel size, $8 \mathrm{~mA}, 90 \mathrm{kVp}, 8.5$ s exposure time and $8 \times 15 \mathrm{FOV})$ and $(350 \mu \mathrm{m}$ voxel size, $10 \mathrm{~mA}, 90 \mathrm{kVp}, 4.5 \mathrm{~s}$ exposure time and $8 \mathrm{x}$ 15 FOV).

\section{Image manipulation}

\section{For 2D measurements:}

One of the axes of the orthogonal planes was used as a reference. The axis level was adjusted to show both markers of distance intended to be measured. On the corresponding image, the 2D ruler was used to measure the distance between the two markers. 


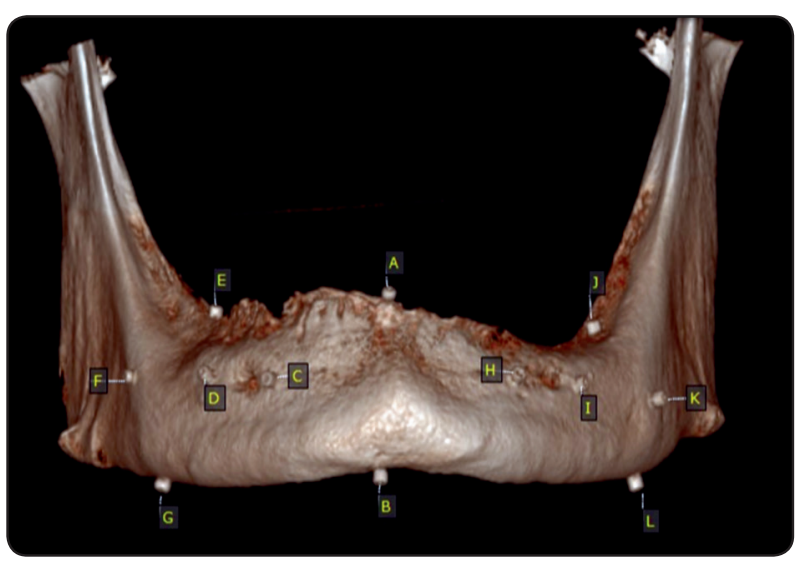

Fig. (1) A 3D rendered CBCT image of one of the mandibles showing the distribution and labeling of the markers on the mandible assigned by letters.

\section{For 3D measurements:}

Using the head orientation in the software, the 3D ruler was used to measure the distance between the centers of the two markers in the form of a straight line. The mandible was rotated in all possible aspects to check the proper position of the terminal points of measurement in relation to corresponding markers.

\section{Statistical Analysis:}

Actual measurements served as the gold standard for all tested methods. The data used was numerical data; so intra- and inter-observer agreement was assessed using Intra-Class Correlation Coefficient
(ICC) (Less than 0.73 is not accepted, 0.73 to $0.9=$ Good, More than 0.94 is Excellent). BlandAltman plot (differences-vs-means plot) was used to show the agreement between two quantitative measurements by studying the mean difference and constructing limits of agreement ${ }^{(8)}$.SPSS $®$ IBM Version 24 and MedCalc ${ }^{\circledR}$ version 12.1 for Windows used for statistical analysis.

\section{RESULTS}

Assessment of intra-observer and inter-observer reliability regarding all measurements showed excellent agreement between the two readings of the first observer. CBCT 2D measurements with voxel size of $250 \mu$ showed a percentage of mean difference $=0.1 \%$ so $95 \%$ percentage of difference was $2.1 \%$ above or $1.9 \%$ below Gold Standard. CBCT 2D measurements with voxel size of $350 \mu$ showed a percentage of mean difference $=0.1 \%$ so $95 \%$ percentage of difference was $1.8 \%$ above or $1.5 \%$ below Gold Standard.CBCT 3D measurements with Voxel Size of $250 \mu$ showed a percentage of mean difference $=0 \%$ so $95 \%$ percentage of difference was $1.6 \%$ below or $1.6 \%$ above Gold Standard. CBCT 3D measurements with Voxel Size of $350 \mu$ showed a percentage of mean difference $=$ $0 \%$ so $95 \%$ percentage of difference was $2.3 \%$ below or $2.3 \%$ above Gold Standard (Table 1).

TABLE (1): Percentage of difference, Limits of agreement and coefficient of variation for different groups compared to Gold standard.

\begin{tabular}{|c|c|c|c|c|c|c|c|c|c|c|c|}
\hline \multirow{2}{*}{ 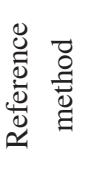 } & \multirow{2}{*}{ Variable } & \multirow{2}{*}{$\mathrm{n}$} & \multicolumn{3}{|c|}{ Differences $\%$} & \multicolumn{4}{|c|}{ Limits of agreement } & \multicolumn{2}{|c|}{$\begin{array}{l}\text { Coefficient of } \\
\text { variation }(\%)\end{array}$} \\
\hline & & & Mean & SD & $95 \% \mathrm{CI}$ & $\begin{array}{l}\text { Lower } \\
\text { limit }\end{array}$ & $95 \% \mathrm{CI}$ & $\begin{array}{c}\text { Upper } \\
\text { limit }\end{array}$ & $95 \% \mathrm{CI}$ & Mean & $95 \% \mathrm{CI}$ \\
\hline \multirow{4}{*}{ 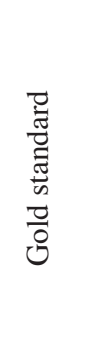 } & 2D $250 \mu$ & 107 & 0.1275 & 1.0225 & $\begin{array}{l}-0.06851 \\
\text { to } 0.3235\end{array}$ & -1.8767 & $\begin{array}{c}-2.2127 \text { to } \\
-1.5407\end{array}$ & 2.1316 & $\begin{array}{c}1.7956 \text { to } \\
2.4676\end{array}$ & 0.7259 & $\begin{array}{c}0.6272 \text { to } \\
0.8247\end{array}$ \\
\hline & 2D $350 \mu$ & 107 & 0.1361 & 0.8616 & $\begin{array}{l}-0.02905 \\
\text { to } 0.3012\end{array}$ & -1.5526 & $\begin{array}{c}-1.8357 \text { to } \\
-1.2695\end{array}$ & 1.8247 & $\begin{array}{c}1.5416 \text { to } \\
2.1078\end{array}$ & 0.6131 & $\begin{array}{c}0.5298 \text { to } \\
0.6965\end{array}$ \\
\hline & $3 \mathrm{D} 250 \mu$ & 107 & -0.00241 & 0.8229 & $\begin{array}{c}-0.1601 \text { to } \\
0.1553\end{array}$ & -1.6153 & $\begin{array}{c}-1.8857 \text { to } \\
-1.3449\end{array}$ & 1.6105 & $\begin{array}{c}1.3401 \text { to } \\
1.8809\end{array}$ & 0.5814 & $\begin{array}{c}0.5024 \text { to } \\
0.6605\end{array}$ \\
\hline & 3D $350 \mu$ & 107 & 0.03197 & 1.2175 & $\begin{array}{c}-0.2014 \text { to } \\
0.2653\end{array}$ & -2.3543 & $\begin{array}{c}-2.7544 \text { to } \\
-1.9543\end{array}$ & 2.4183 & $\begin{array}{c}2.0182 \text { to } \\
2.8183\end{array}$ & 0.8282 & $\begin{array}{c}0.7156 \text { to } \\
0.9410\end{array}$ \\
\hline
\end{tabular}




\section{DISCUSSION}

The mandibles included in our study were free from fractures or pathological lesions so they wouldn't affect the linear measurements recorded by the caliper and those taken on the software, as fracture lines might interfere with the measurements sites causing difficulty in determining the outline of the selected slice. Also pathologies might affect the continuity of the mandible surfaces or cortices which will cause inability to mark the measurement sites.

Moreover, the mandibles were immersed in water to simulate the soft tissue, approximating the clinical situation. The mandibles were immersed in water inside a plastic box to facilitate positioning of the mandibles in the FOV of the machine and adjustment of the mid-sagittal plane (reference line of the machine) with the midline of the mandibles.

Different voxel sizes were used to investigate if larger voxel sizes could affect the accuracy of linear measurements. The voxel sizes of $0.250 \mathrm{~mm}$ and $0.350 \mathrm{~mm}$ were used as they are commonly used in implantology and detection of maxillofacial pathologies.

Regarding 2D measurements, among the studies that yielded results similar to ours was Ganguly et al. 2016 ${ }^{(7)}$. However, they used four cadaver heads (maxilla and mandible), unlike our methodology in which we used only mandibles. They used 3 scanning protocols: large FOV/0.3 mm voxel, large FOV/0.2 mm voxel and small FOV/0.16 mm voxel for height and width of alveolar ridge. They found no statistical difference among protocols and that CBCT measurements are accurate to measure the height and width of alveolar bone. They concluded that smaller voxel sizes do not result in greater accuracy of linear measurements, which is in accordance with our study.

Also, Gorucu-coskuner and El, 2019(9) found that 2D linear TMJ measurements in the coronal and sagittal views were reliable and accurate, which goes in line with our results.

However, contradictory to our results, Elshenawy et al. 2019(5) found that increasing the voxel size along with increasing the FOV could adversely affect the accuracy of linear measurements particularly if small distances are to be assessed. This could be attributed to the differences in the technical parameters they used and not using soft tissue simulation.

Regarding 3D measurements, in accordance with our results, Kamburoglu et al. 2011 ${ }^{(13)}$ found that $\mathrm{CBCT}$ image measurements were identical and highly correlated with digital caliper measurements. Our results were comparable to theirs which showed that increasing voxel resolution did not affect the accuracy of surface model measurements.

Contradictory to our results, however, another study which measured 13 sites (3D) on human dry skulls found that CBCT images underestimated the real distances between skull sites. However, the differences between CBCT and direct measurements were only statistically significant for measurements of the skull base ${ }^{(15)}$. These differences between that study findings and ours can be related to the observer performance and calibration or hardware/software of the different systems tested. Also they used 2-mm diameter metal spheres fixed to the skull to mark the distances to be measured, which might have caused scatter and beam hardening that adversely affected observer performance.

In conclusion, 2D and 3D measurements driven from CBCT imaged with voxel size of $350 \mu$ are accurate as measurements driven from $\mathrm{CBCT}$ imaged with voxel size of $250 \mu$ with less radiation delivered to the patient. Also 3D measurements driven from CBCT are accurate as 2D measurements. Finally, 2D and 3D CBCT measurements driven from $\mathrm{CBCT}$ are reliable. 


\section{REFERENCES}

1. Anderson, P. J., Yong, R., Surman, T. L., Rajion, Z. A., \& Ranjitkar, S. (2014). Application of three-dimensional computed tomography in craniofacial clinical practice and research. Aust Dent J., 59(SUPPL. 1), 174-185.

2. Bernardes, R. A., de Moraes, I. G., Húngaro Duarte, M. A., Azevedo, B. C., de Azevedo, J. R., \& Bramante, C. M. (2009). Use of cone-beam volumetric tomography in the diagnosis of root fractures. Oral Surg Oral Med Oral Pathol Oral RadiolEndod.,108(2): 270-277.

3. Blessmann, M., Pohlenz, P., Blake, F. A. S., Lenard, M., Schmelzle, R., \&Heiland, M. (2007). Validation of a new training tool for ultrasound as a diagnostic modality in suspected midfacial fractures. Int J Oral Maxillofac Surg., 36(6): 501-506.

4. Damstra, J., Fourie, Z., Huddleston Slater, J. J. R., \&Ren, Y. (2010). Accuracy of linear measurements from conebeam computed tomography-derived surface models of different voxel sizes. Am J OrthodDentofacialOrthop., 137(1): 16.e1-16.e6.

5. Elshenawy, H., Aly, W., Salah, N., Nasry, S., Anter, E., \&Ekram, K. (2019). Influence of Small, Midi, Medium and Large Fields of View on Accuracy of Linear Measurements in CBCT Imaging: Diagnostic Accuracy Study. Open Access Maced J Med Sci., 7(6): 1037-1041.

6. Fernandes, T.M.F.,Adamczyk, J., Poleti, M.L., Henriques, J. F, C., Friedland, Garib, D. G. (2015). Comparison between $3 \mathrm{D}$ volumetric rendering and multiplanar slices on the reliability of linear measurements on CBCT images : an in vitro study. J Appl Oral Sci., 23(1): 56-63.

7. Ganguly, R., Ramesh, A., \&Pagni, S. (2016). The accuracy of linear measurements of maxillary and mandibular edentulous sites in conebeam computed tomography images with different fields of view and voxel sizes under simulated clinical conditions. Imaging Sci Dent., 46(2), 93-101.

8. Giavarina, D. (2015). Understanding Bland Altman analysis. Biochem Med., 25(2): 141-151.

9. Gorucu-Coskuner, H., Atik, E., \& El, H. (2019). Reliability of cone-beam computed tomography for temporomandibular joint analysis. Korean J Orthod.,49(2): 81-88.

10. Guerrero, M. E., Noriega, J., Castro, C., \& Jacobs, R. (2014). Does cone-beam CT alter treatment plans? Comparison of preoperative implant planning using panoramic versus cone-beam CT images.Imaging Sci Dent., 44(2): 121-128.
11. Hassan, B., Metska, M. E., Ozok, A. R., van der Stelt, P., \& Wesselink, P. R. (2009). Detection of Vertical Root Fractures in Endodontically Treated Teeth by a Cone Beam Computed Tomography Scan. J Endod.,35(5): 719-722.

12. Hassan, B., Metska, M. E., Ozok, A. R., van der Stelt, P., \&Wesselink, P. R. (2010). Comparison of five cone beam computed tomography systems for the detection of vertical root fractures. J Endod.,36(1): 126-129.

13. Kamburoglu, K., Kolsuz, E., Kurt, H., Kiliç, C., Özen, T., \& Paksoy, C.S. (2011). Accuracy of CBCT measurements of a human skull. J Digit Imaging., 24(5): 787-793.

14. Kasaj, A., \&Willershausen, B. (2007). Digital volume tomography for diagnostics in periodontology. Int $\mathrm{J}$ Comput Dent., 10(2): 155-168.

15. Lascala, C. A., Panella, J., \& Marques, M. M. (2004). Analysis of the accuracy of linear measurements obtained by cone beam computed tomography (CBCT-NewTom). DentomaxillofacRadiol., 33(5): 291-294.

16. Lennon, S., Patel, S., Foschi, F., Wilson, R., Davies, J., \& Mannocci, F. (2011). Diagnostic accuracy of limitedvolume cone-beam computed tomography in the detection of periapical bone loss: $360^{\circ}$ scans versus $180^{\circ}$ scans. IntEndod J., 44(12): 1118-1127.

17. Madani, Z., Moudi, E., Bijani, A., \&Mahmoudi, E. (2016). Diagnostic accuracy of cone-beam computed tomography and periapical radiography in internal root resorption. Iran Endod J., 11(1): 51-56.

18. Miracle, A.C., \&Mukherji, S. K. (2009). Conebeam CT of the Head and Neck, Part 1: Physical Principles. AJNR Am J Neuroradiol.,30(6): 1088-1095.

19. Moshfeghi, M., Tavakoli, M. A., Hosseini, E. T., Hosseini, A. T., \&Hosseini, I. T. (2012). Analysis of linear measurement accuracy obtained by cone beam computed tomography (CBCT-NewTom VG). Dent Res J., 9(Suppl 1), S57-62.

20. Waltrick, K. B., de Abreu Junior, M. J. N., Corrêa, M., Zastrow, M. D., \&D'Avila Dutra, V. (2012). Accuracy of linear measurements and visibility of the mandibular canal of cone-beam computed tomography images with different voxel sizes: an in vitro study. J Periodontol.,84(1): 68-77.

21. Zizelmann, C., Gellrich, N. C., Metzger, M. C., Schoen, R., Schmelzeisen, R., \& Schramm, A. (2007). Computerassisted reconstruction of orbital floor based on cone beam tomography. Br J Oral Maxillofac Surg., 45(1): 79-80. 